\title{
FULLY COUPLED FBSDE WITH BROWNIAN MOTION AND POISSON PROCESS IN STOPPING TIME DURATION
}

\author{
ZHEN WU \\ (Received 7 October 2000; revised 18 January 2002)
}

Communicated by V. Stefanov

\begin{abstract}
We first give the existence and uniqueness result and a comparison theorem for backward stochastic differential equations with Brownian motion and Poisson process as the noise source in stopping time (unbounded) duration. Then we obtain the existence and uniqueness result for fully coupled forwardbackward stochastic differential equation with Brownian motion and Poisson process in stopping time (unbounded) duration. We also proved a comparison theorem for this kind of equation.
\end{abstract}

2000 Mathematics subject classification: primary 60H10, $60 \mathrm{G} 40$.

Keywords and phrases: stochastic differential equations, stopping time, random measure, Poisson process, comparison theorem.

\section{Introduction}

Nonlinear backward stochastic differential equations with Brownian motion as noise sources (BSDE in short) have been independently introduced by Pardoux and Peng [11] and Duffie and Epstein [4]. It was soon discovered by Peng [13] that, coupled with a forward stochastic differential equation (SDE in short), such BSDE give a probabilistic interpretation for a large kind of second order quasilinear partial differential equations (PDE in short). In this paper Peng also gave an existence and uniqueness result of BSDE in stopping time duration which can take infinite value. And then Darling and Pardoux [3] proved an existence and uniqueness result for BSDE in stopping time under different assumptions. They applied their result to construct a continuous viscosity solution for a class of semilinear elliptic PDE. In [8], El Karoui, Peng and

This work is supported by Chinese National Natural Science Foundation (Grant No. 10001022), the Excellent Young Teachers Program and the Doctoral Program Foundation of MOE, P.R.C.

(C) 2003 Australian Mathematical Society $1446-7887 / 03 \$ A 2.00+0.00$ 
Quenez gave a comparison theorem to BSDE and some applications in optimal control and financial mathematics.

Fully coupled forward-backward stochastic differential equations with Brownian motion (FBSDE in short) can be encountered in the optimization problem when applying stochastic maximum principle and mathematical finance considering large investor in security market. Antonelli [1] first studied this kind of equations and obtained the local existence and uniqueness results, that is, the time duration on which the solutions exist (without explosion) has to be sufficiently small. He also gave a counterexample to show that the Lipschitz condition is not enough for the existence of FBSDE in an arbitrarily large time duration. Using PDE method, Ma, Protter and Yong [9] successfully obtained the existence and uniqueness result for an arbitrarily prescribed time duration. But they needed the forward SDE to be nondegenerate and the coefficients not to be randomly disturbed. Using probability method, Hu and Peng [6] obtained the existence and uniqueness result when forward and backward equations take same dimensions under some monotone assumptions. Hamadène [5] weaken their monotone assumptions and discussed the application in stochastic differential games. Peng and the author [17] extend their results to different dimensional FBSDE and weaken the monotone assumptions so that the results can be used widely. The main method is to introduce an $m \times n$ full rank matrix $G$ to overcome the difficulty of the different dimensions. Yong [21] made the above method systematic and called it 'continuation method'. In [12], Pardoux and Tang also gave the existence and uniqueness results for FBSDE under some monotone conditions different from [6] and [17]. Recently, Peng and Shi [16] gave an existence and uniqueness result of FBSDE with infinite horizon. But the solution is in a square integrable space, the infinite time value of the solution must be zero.

The BSDE with Poisson process (BSDEP in short) was first discussed by Tang and $\mathrm{Li}$ [19]. The stochastic process in the equation is discontinuous with random jump. After then Situ Rong [18] obtained an existence and uniqueness result with non-Lipschitz coefficients for BSDEP. Using this kind of BSDEP Barles, Buckdahn and Pardoux [2] gave the probabilistic interpretation for a system of parabolic integropartial differential equation and proved that there exists a unique viscosity solution for this kind of PDE systems. In Section 2 we study the BSDEP in stopping time duration, here the stopping time is unbounded and can take infinite value. Under a Lipschitz condition suitable for our case, we get the existence and uniqueness result for BSDEP using fixed point principle and other technique. Further in Section 2, we give a comparison theorem for BSDEP in stopping time. The conclusion is similar with that in [8]. We only need to control the height of the jump in BSDEP.

In Section 3, we consider fully coupled forward-backward stochastic differential equations with Brownian motion and Poisson process (FBSDEP in short) in stopping time duration. Suitable for the case that the stopping time can be infinite, we prove 
an existence and uniqueness result under a Lipschitz and monotone assumptions, the infinite time value of the solution not necessarily be required zero.

In Section 4, we give a comparison theorem for FBSDEP in stopping time. The idea in the proof is to use duality technique and stopping time technique. The duality technique is usually used in optimal control theory to introduce the adjoint equation for proving the maximum principle (see $[14,20]$ ). Another technique is to analyze the jump height under the limit assumption. This kind of comparison theorem can be used to connect FBSDEP with a parabolic integro-PDE system and study the existence of the viscosity solution for this PDE system. The PDE system form should be a PDE combined by the algebra equation. For no jump case this kind of PDE form can be seen in [15]. Here the comparison theorem of FBSDEP is established only at time 0 , we cannot get the result in the whole random interval. We also give a counterexample to show this point.

\section{BSDEP in stopping time duration}

Let $\left(\Omega, \mathscr{F},\left\{\mathscr{F}_{1}\right\}_{t \geq 0}, P\right)$ be a stochastic basis such that $\mathscr{F}_{0}$ contains all $P$-null elements of $\mathscr{F}$ and $\mathscr{F}_{t+}=\bigcap_{\epsilon>0} \mathscr{F}_{t+\epsilon}=\mathscr{F}_{t}, t \geq 0$. We suppose that the filtration $\left\{\mathscr{F}_{t}\right\}_{t \geq 0}$ is generated by the following two mutually independent processes:

- a $d$-dimensional standard Brownian motion $\left\{B_{t}\right\}_{t \geq 0}$ and

- a Poisson random measure $N$ on $\mathbb{R}_{+} \times Z$, where $Z \subset \mathbb{R}^{l}$ is nonempty open set equipped with its Borel field $\mathscr{B}(\mathscr{Z})$, with compensator $\widehat{N}(d z, d t)=n(d z) d t$, such that $\widetilde{N}(A \times[0, t])=(N-\widehat{N})(A \times[0, t])_{t \geq 0}$ is a martingale for all $A \in \mathscr{B}(\mathscr{Z})$ satisfying $n(A)<\infty$. $n$ is assumed to be a $\sigma$-finite measure on $(Z, \mathscr{B}(\mathscr{Z}))$ and called the characteristic measure. $\mathscr{F}_{\infty}=\bigvee_{t \geq 0} \mathscr{F}_{t}$. Let $\tau=\{\tau(\omega)\}$ be $\mathscr{F}_{t}$ stopping time and take value in $[0, \infty]$. We introduce the following notations:

$$
\begin{aligned}
& \mathscr{S}^{2}=\left\{v_{t}, 0 \leq t \leq \tau \text {, is a } \mathscr{F}_{t} \text { adapted process such that } \mathbb{E}\left[\sup _{0 \leq t \leq \tau}\left|v_{t}\right|^{2}\right]<\infty\right\}, \\
& \mathscr{H}^{2}=\left\{v_{t}, 0 \leq t \leq \tau \text {, is a } \mathscr{F}_{t} \text { adapted process such that } \mathbb{E}\left[\int_{0}^{\tau}\left|v_{t}\right|^{2} d t\right]<\infty\right\} \text {, } \\
& L^{2}=\left\{\xi, \xi \text { is a } \mathscr{F}_{\tau} \text { measurable random variable such that } \mathbb{E}|\xi|^{2}<\infty\right\}, \\
& F_{N}^{2}=\left\{k_{t}(\cdot), 0 \leq t \leq \tau \text {, is a } \mathscr{F}_{t}\right. \text { predictable process such that } \\
& \left.\mathbb{E}\left[\int_{0}^{\tau} \int_{Z}\left|k_{t}(z)\right|^{2} n(d z) d t\right]<\infty\right\} \text {. }
\end{aligned}
$$

We consider the following BSDEP in stopping time duration

$$
p_{t}=\xi+\int_{t \wedge \tau}^{\tau} f\left(s, p_{s}, q_{s}, k_{s}\right) d s-\int_{t \wedge \tau}^{\tau} q_{s} d B_{s}-\int_{t \wedge \tau}^{\tau} \int_{Z} k_{s_{-}}(z) \tilde{N}(d z d s),
$$

where $t \geq 0, \xi \in L^{2}$ and $f$ is a map from $\Omega \times[0, \infty] \times \mathbb{R}^{m} \times \mathbb{R}^{m \times d} \times \mathbb{R}^{m}$ onto $\mathbb{R}^{m}$ which satisfies 
(H2.1) For every $(p, q, k) \in \mathbb{R}^{m+m \times d+m}, f(\cdot, p, q, k)$ is progressively measurable and $\mathbb{E}\left(\int_{0}^{\infty}|f(s, 0,0,0)| d s\right)^{2}<\infty$.

(H2.2) There exist three positive deterministic functions $u_{1}(t), u_{2}(t)$ and $u_{3}(t)$, such that $\forall\left(p^{i}, q^{i}, k^{i}\right), i=1,2$,

$$
\begin{aligned}
& \left|f\left(t, p^{1}, q^{1}, k^{1}\right)-f\left(t, p^{2}, q^{2}, k^{2}\right)\right| \\
& \quad \leq u_{1}(t)\left|p^{1}-p^{2}\right|+u_{2}(t)\left|q^{1}-q^{2}\right|+u_{3}(t)\left|k^{1}-k^{2}\right|, \quad t \geq 0,
\end{aligned}
$$

and $\int_{0}^{\infty} u_{1}(t) d t<\infty, \int_{0}^{\infty} u_{2}^{2}(t) d t<\infty, \int_{0}^{\infty} u_{3}^{2}(t) d t<\infty$.

Then we have

THEOREM 2.1. Assume $\xi \in L^{2}$ and $f$ satisfies (H2.1)-(H2.2), then there exists a unique solution $(p, q, k) \in \mathscr{S}^{2} \times \mathscr{H}^{2} \times F_{N}^{2}$ satisfying the $\operatorname{BSDEP}(2.1)$.

Proof. For the uniqueness, let $(\bar{p}, \bar{q}, \bar{k})$ be another solution, we set $\hat{p}=(p-\bar{p})$, $\hat{q}=(q-\bar{q}), \hat{k}=(k-\bar{k})$. Using Itô's formula to $\left|\hat{p}_{t}\right|^{2}$, similarly with the proof in [11] for fixed time $T$ without jump except the Lipschitz constants being replaced by $u_{1}(t), u_{2}(t)$ and $u_{3}(t), t \geq 0$, we can get the conclusion from the assumption (H2.2) and Gronwall's lemma.

For the existence we want to construct one contraction map for (2.1) and get the solution. However, the stopping time duration is unbounded and can be infinite, so we cannot get this in one step. We divide the proof into two steps.

First step. Assume

$$
\left(\int_{0}^{\infty} u_{1}(t) d t\right)^{2}+\int_{0}^{\infty} u_{2}^{2}(t) d t+\int_{0}^{\infty} u_{3}^{2}(t) d t<\frac{1}{15}
$$

For every $(p, q, k) \in \mathscr{S}^{2} \times \mathscr{H}^{2} \times F_{N}^{2}$, we have

$$
\begin{aligned}
& \mathbb{E}\left[\xi+\int_{0}^{\tau} f\left(t, p_{t}, q_{t}, k_{t}\right) d t\right]^{2} \\
& \quad \leq \mathbb{E}\left[|\xi|+\int_{0}^{\tau}\left(|f(t, 0,0,0)|+u_{1}(t)\left|p_{t}\right|+u_{2}(t)\left|q_{t}\right|+u_{3}(t)\left|k_{t}\right|\right) d t\right]^{2}
\end{aligned}
$$

and

$$
\begin{aligned}
& \mathbb{E}\left(\int_{0}^{\tau} u_{1}(t)\left|p_{t}\right| d t\right)^{2} \leq\left(\int_{0}^{\infty} u_{1}(t) d t\right)^{2}\|p(\cdot)\|_{\mathscr{S}^{2}}^{2}<\infty, \\
& \mathbb{E}\left(\int_{0}^{\tau} u_{2}(t)\left|q_{t}\right| d t\right)^{2} \leq\left(\int_{0}^{\infty} u_{2}^{2}(t) d t\right)\|q(\cdot)\|_{\mathscr{H}^{2}}^{2}<\infty, \\
& \mathbb{E}\left(\int_{0}^{\tau} u_{3}(t)\left|k_{t}\right| d t\right)^{2} \leq\left(\int_{0}^{\infty} u_{3}^{2}(t) d t\right)\|k(\cdot)\|_{F_{N}^{2}}^{2}<\infty
\end{aligned}
$$




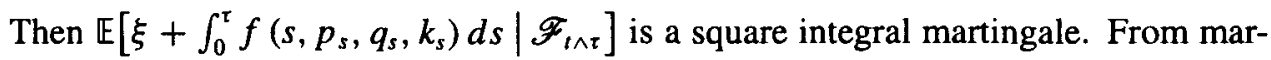
tingale representation theorem, there exists $\left(Q_{s}, K_{s}\right)$ satisfying

$$
\begin{aligned}
& \mathbb{E}\left[\xi+\int_{0}^{\tau} f\left(s, p_{s}, q_{s}, k_{s}\right) d s \mid \mathscr{F}_{\iota \wedge \tau}\right] \\
& \quad=\mathbb{E}\left[\xi+\int_{0}^{\tau} f\left(s, p_{s}, q_{s}, k_{s}\right) d s\right]+\int_{0}^{t \wedge \tau} Q_{s} d B_{s}+\int_{0}^{t \wedge \tau} \int_{z} K_{s_{-}}(z) \tilde{N}(d z d s) .
\end{aligned}
$$

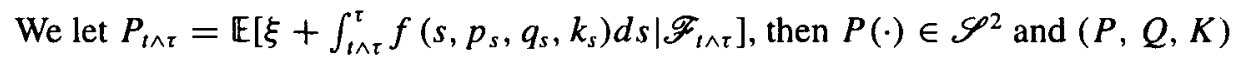
is the solution of the BSDEP

$$
P_{t \wedge \tau}=\xi+\int_{t \wedge \tau}^{\tau} f\left(s, p_{s}, q_{s}, k_{s}\right) d s-\int_{t \wedge \tau}^{\tau} Q_{s} d B_{s}-\int_{t \wedge \tau}^{\tau} \int_{Z} K_{s_{-}}(z) \tilde{N}(d z d s)
$$

This equation introduces the map $\Phi: \mathscr{S}^{2} \times \mathscr{H}^{2} \times F_{N}^{2} \rightarrow \mathscr{S}^{2} \times \mathscr{H}^{2} \times F_{N}^{2}$ by $\Phi:(p, q, k) \rightarrow(P, Q, K)$. We use the following method, which is similar with that in [1], to get the solution of BSDE in $L^{1}$ space within the fixed time duration, to prove the above map is a contraction. Let $\Phi:\left(p^{i}, q^{i}, k^{i}\right) \rightarrow\left(P^{i}, Q^{i}, K^{i}\right), i=1,2$, $\widehat{P}=P^{1}-P^{2}, \widehat{Q}=Q^{1}-Q^{2}, \widehat{K}=K^{1}-K^{2}, \hat{p}=p^{1}-p^{2}, \hat{q}=q^{1}-q^{2}, \hat{k}=k^{1}-k^{2}$, $\hat{f_{s}}=f\left(s, p^{1}, q^{1}, k^{1}\right)-f\left(s, p^{2}, q^{2}, k^{2}\right)$. From Doob's inequality,

$$
\begin{gathered}
\|\widehat{P}(\cdot)\|_{\mathscr{S}^{2}}^{2}=\mathbb{E} \sup _{0 \leq t \leq \tau}\left|\mathbb{E}\left(\int_{t}^{\tau} \hat{f_{s} d s \mid} \mathscr{F}_{l}\right)\right|^{2} \leq 4 \mathbb{E}\left(\int_{0}^{\tau}\left|\hat{f_{s}}\right| d s\right)^{2}, \\
\|\widehat{Q}(\cdot)\|_{\mathscr{H}^{2}}^{2}+\|\widehat{K}(\cdot)\|_{F_{N}^{2}}^{2}=\mathbb{E}\left|\int_{0}^{\tau} \hat{f_{s} d s}\right|^{2}-\left|\mathbb{E} \int_{0}^{\tau} \hat{f_{s} d s}\right|^{2} \leq \mathbb{E}\left(\int_{0}^{\tau}\left|\hat{f_{s}}\right| d s\right)^{2} .
\end{gathered}
$$

We note that $\mathscr{B}^{2}=\mathscr{S}^{2} \times \mathscr{H}^{2} \times F_{N}^{2}$. So

$$
\begin{aligned}
\left\|\Phi\left(p^{1}, q^{1}, k^{1}\right)-\Phi\left(p^{2}, q^{2}, k^{2}\right)\right\|_{\mathscr{B}^{2}}^{2} \\
=\|\widehat{P}(\cdot)\|_{\mathscr{S}^{2}}^{2}+\|\widehat{Q}(\cdot)\|_{\mathscr{H}^{2}}^{2}+\|\widehat{K}(\cdot)\|_{F_{N}^{2}}^{2} \leq 5 \mathbb{E}\left(\int_{0}^{t}\left|\hat{f_{s}}\right| d s\right)^{2} \\
\leq 15\left[\left(\int_{0}^{\infty} u_{1}(t) d t\right)^{2}+\int_{0}^{\infty} u_{2}^{2}(t) d t+\int_{0}^{\infty} u_{3}^{2}(t) d t\right] \\
\times\left[\|\hat{p}(\cdot)\|_{\mathscr{S}^{2}}^{2}+\|\hat{q}(\cdot)\|_{\mathscr{H}^{2}}^{2}+\|\hat{k}(\cdot)\|_{F_{N}^{2}}^{2}\right] .
\end{aligned}
$$

From the assumption $\left(\int_{0}^{\infty} u_{1}(t) d t\right)^{2}+\int_{0}^{\infty} u_{2}^{2}(t) d t+\int_{0}^{\infty} u_{3}^{2}(t) d t<1 / 15$, then $\Phi: \mathscr{B}^{2} \rightarrow \mathscr{B}^{2}$ is a strict contraction, BSDEP (2.1) has one unique solution.

Second step. Assume $\int_{0}^{\infty} u_{1}(t) d t<\infty, \int_{0}^{\infty} u_{2}^{2}(t) d t<\infty, \int_{0}^{\infty} u_{3}^{2}(t) d t<\infty$. Then there exists $T>0$, such that $\left(\int_{T}^{\infty} u_{1}(t) d t\right)^{2}+\int_{T}^{\infty} u_{2}^{2}(t) d t+\int_{T}^{\infty} u_{3}^{2}(t) d t<1 / 15$. We let $f_{1}(s, p, q, k)=I_{[r, \infty]}(t) f(t, p, q, k)$, then $f_{1}$ satisfies Lipschitz condition 
$(\mathrm{H} 2.2)$ with $\bar{u}_{1}(t)=I_{[T, \infty]}(t) u_{1}(t), \bar{u}_{2}(t)=I_{[T, \infty]}(t) u_{2}(t), \bar{u}_{3}(t)=I_{[T, \infty]}(t) u_{3}(t)$ and $\left(\int_{0}^{\infty} \bar{u}_{1}(t) d t\right)^{2}+\int_{0}^{\infty} \bar{u}_{2}^{2}(t) d t+\int_{0}^{\infty} \bar{u}_{3}^{2}(t) d t<1 / 15$. So there exists solution $(\tilde{p}(\cdot), \tilde{q}(\cdot), \tilde{k}(\cdot))$ from the first step, such that, for $t \geq T$,

$$
\tilde{p}_{t \wedge \tau}=\xi+\int_{t \wedge \tau}^{\tau} f_{1}\left(s, \tilde{p}_{s}, \tilde{q}_{s}, \tilde{k}_{s}\right) d s-\int_{t \wedge \tau}^{\tau} \tilde{q}_{s} d B_{s}-\int_{t \wedge \tau}^{\tau} \int_{Z} \tilde{k}_{s_{-}}(z) \tilde{N}(d z d s) .
$$

Then we consider the following BSDEP,

$$
\bar{p}_{t}=\tilde{p}_{T \wedge \tau}+\int_{t}^{T \wedge \tau} f\left(s, \bar{p}_{s}, \bar{q}_{s}, \bar{k}_{s}\right) d s-\int_{t}^{T \wedge \tau} \bar{q}_{s} d B_{s}-\int_{t}^{T \wedge \tau} \int_{Z} \bar{k}_{s_{-}}(z) \tilde{N}(d z d s)
$$

$t \in[0, T \wedge \tau]$. From the result in [10] or the result for fixed time in [19], which only need minor change suitable for our case, there exists unique solution $(\bar{p}, \bar{q}, \bar{k})$. Let us set $p_{t}=I_{[0, T \wedge \tau]}(t) \bar{p}_{t}+I_{(T \wedge \tau, \tau]} \tilde{p}_{t}, q_{t}=I_{[0, T \wedge \tau]}(t) \bar{q}_{t}+I_{(T \wedge \tau, \tau]} \tilde{q}_{t}, k_{t}=I_{[0, T \wedge \tau]}(t) \bar{k}_{t}+$ $I_{(T \wedge \tau, \tau]} \widetilde{k}_{t}$, it is easy to check that this is a solution of $\operatorname{BSDEP}(2.1)$. The proof is completed.

Similarly to the comparison theorem of BSDE in [8], we will give this kind of theorem for BSDEP in stopping time in the remaining part of this section. But the appearance of jump process needs one new condition to limit the height of the jump besides the Lipschitz condition in (H2.2).

We consider the following two BSDEPs in stopping time, here $m=1$.

$$
p_{i}^{i}=\xi^{i}+\int_{I \wedge \tau}^{\tau} f^{i}\left(s, p_{s}^{i}, q_{s}^{i}, k_{s}^{i}\right) d s-\int_{t \wedge \tau}^{\tau} q_{s}^{i} d B_{s}-\int_{l \wedge \tau}^{\tau} \int_{Z} k_{s_{-}}^{i}(z) \tilde{N}(d z d s),
$$

where $i=1,2, \xi^{i} \in L^{2}, f^{i}$ satisfy (H2.1) and (H2.2). From Theorem 2.1, there exist $\left(p^{i}(\cdot), q^{i}(\cdot), k^{i}(\cdot)\right) \in \mathscr{S}^{2} \times \mathscr{H}^{2} \times F_{N}^{2}$ which satisfy BSDEP (2.5) respectively. We also assume

(H2.3) $\xi^{1} \geq \xi^{2}, f^{1}\left(s, p^{2}, q^{2}, k^{2}\right) \geq f^{2}\left(s, p^{2}, q^{2}, k^{2}\right), s \geq 0$.

(H2.4) $-c_{2}(s)<\left(f^{1}\left(s, p^{2}, q^{2}, k^{1}\right)-f^{2}\left(s, p^{2}, q^{2}, k^{2}\right)\right) /\left(k^{1}-k^{2}\right)<c_{1}(s)$, when $k^{1}-k^{2} \neq 0, c_{1}(s)$ and $c_{2}(s)$ are two positive deterministic functions which satisfy $\int_{0}^{\infty} c_{1}(s) d s<\infty, \int_{0}^{\infty} c_{2}(s) d s<\infty$ and $c_{2}(s)<1, s \geq 0$.

Then we have

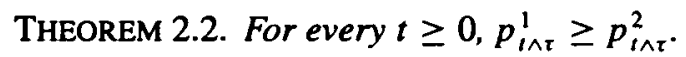

The proof is almost the same as the proof of the comparison theorem [8, Theorem 2.2] for BSDE without jump. We omit it.

When $\tau \leq T<\infty$, we can take $u_{1}(t), u_{2}(t)$ and $u_{3}(t), 0 \leq t \leq T$, to be constants, then the result of BSDEP in bounded time duration is the special case of our result in this section. 


\section{Existence and uniqueness of FBSDEP in stopping time duration}

In this section, we discuss the fully coupled FBSDEP in stopping time duration. We consider

$$
\left\{\begin{aligned}
x_{t}= & a+\int_{0}^{t \wedge \tau} b\left(s, x_{s}, p_{s}, q_{s}, k_{s}\right) d s+\int_{0}^{t \wedge \tau} \sigma\left(s, x_{s}, p_{s}, q_{s}, k_{s}\right) d B_{s} \\
& +\int_{0}^{\imath \wedge \tau} \int_{z} g\left(s_{-}, x_{s_{-}}, p_{s_{-}}, q_{s_{-}}, k_{s_{-}}(z), z\right) \tilde{N}(d z d s), \\
p_{t \wedge \tau}= & \Phi\left(x_{\tau}\right)+\int_{t \wedge \tau}^{\tau} f\left(s, x_{s}, p_{s}, q_{s}, k_{s}\right) d s-\int_{t \wedge \tau}^{\tau} q_{s} d B_{s} \\
& -\int_{0}^{t \wedge \tau} \int_{Z} k_{s_{-}}(z) \tilde{N}(d z d s) .
\end{aligned}\right.
$$

Here $t>0,(x, p, q, k)$ take value in $\mathbb{R}^{m} \times \mathbb{R}^{m} \times \mathbb{R}^{m \times d} \times \mathbb{R}^{m}$,

$$
\begin{aligned}
& b: \Omega \times[0, \infty] \times \mathbb{R}^{m} \times \mathbb{R}^{m} \times \mathbb{R}^{m \times d} \times \mathbb{R}^{m} \rightarrow \mathbb{R}^{m}, \\
& \sigma: \Omega \times[0, \infty] \times \mathbb{R}^{m} \times \mathbb{R}^{m} \times \mathbb{R}^{m \times d} \times \mathbb{R}^{m} \rightarrow \mathbb{R}^{m \times d}, \\
& g: \Omega \times[0, T] \times \mathbb{R}^{m} \times \mathbb{R}^{m} \times \mathbb{R}^{m \times d} \times \mathbb{R}^{m} \times Z \rightarrow \mathbb{R}^{m}, \\
& f: \Omega \times[0, T] \times \mathbb{R}^{m} \times \mathbb{R}^{m} \times \mathbb{R}^{m \times d} \times \mathbb{R}^{m} \rightarrow \mathbb{R}^{m}, \quad \Phi: \Omega \times \mathbb{R}^{m} \rightarrow \mathbb{R}^{m} .
\end{aligned}
$$

We assume the following:

(H3.1) For every $(x, p, q, k) \in \mathbb{R}^{m+m+m \times d+m}, \Phi(x) \in L^{2}, b, \sigma, g$ and $f$ are progressively measurable and

$$
\begin{aligned}
& \mathbb{E}\left(\int_{0}^{\infty}|b(s, 0,0,0,0)| d s\right)^{2}+\mathbb{E}\left(\int_{0}^{\infty}|f(s, 0,0,0,0)| d s\right)^{2} \\
& \quad+\mathbb{E} \int_{0}^{\infty}|\sigma(s, 0,0,0,0)|^{2} d s+\mathbb{E} \int_{0}^{\infty} \int_{z}|g(s, 0,0,0,0, z)|^{2} n(d z) d s<\infty .
\end{aligned}
$$

(H3.2) There exists a positive deterministic bounded function $u_{1}(t)$, such that for every $\left(x^{i}, p^{i}, q^{i}, k^{i}\right) \in \mathbb{R}^{m+m+m \times d+m}, i=1,2$,

$$
\begin{aligned}
& \left|l\left(t, x^{1}, p^{1}, q^{1}, k^{1}\right)-l\left(t, x^{2}, p^{2}, q^{2}, k^{2}\right)\right| \\
& \quad \leq u_{1}(t)\left[\left|x^{1}-x^{2}\right|+\left|p^{1}-p^{2}\right|+\left|q^{1}-q^{2}\right|+\left|k^{1}-k^{2}\right|\right], \quad t \geq 0
\end{aligned}
$$

$l=b, \sigma, f, g$ respectively, and $\int_{0}^{\infty} u_{1}(t) d t<\infty, \int_{0}^{\infty} u_{1}^{2}(t) d t<\infty$. There exists a constant $C>0$ such that $\left|\Phi\left(x_{1}\right)-\Phi\left(x_{2}\right)\right| \leq C\left|x_{1}-x_{2}\right|$.

We introduce the notations

$$
u=\left(\begin{array}{c}
x \\
p \\
q \\
k
\end{array}\right), \quad A(t, u)=\left(\begin{array}{c}
-f \\
b \\
\sigma \\
g
\end{array}\right)(t, u)
$$


where $\sigma=\left(\sigma_{1} \cdots \sigma_{d}\right)$. We use the usual inner product and Euclidean norm in $\mathbb{R}^{m}$, $\mathbb{R}^{m \times d}$ and assume the following monotone assumptions:

(H3.3) For every $u=(x, p, q, k), \bar{u}=(\bar{x}, \bar{p}, \bar{q}, \bar{k}), \hat{u}=(\hat{x}, \hat{p}, \hat{q}, \hat{k})=(x-\bar{x}$, $p-\bar{p}, q-\bar{q}, k-\bar{k})$,

$$
\left\{\begin{aligned}
\langle A(t, u)-A(t, \bar{u}), \hat{u}\rangle & \leq-\beta_{1} u_{1}(t)|\hat{x}|^{2}-\beta_{2} u_{1}(t)\left(|\hat{p}|^{2}+|\hat{q}|^{2}+|\hat{k}|^{2}\right), \\
\langle\Phi(x)-\Phi(\bar{x}), x-\bar{x}\rangle & \geq \mu_{1}|\hat{x}|^{2},
\end{aligned}\right.
$$

where $\beta_{1}, \beta_{2}$ and $\mu_{1}$ are given nonnegative constants with $\beta_{1}+\beta_{2}>0, \mu_{1}+\beta_{2}>0$.

REMARK 3.1. (i) For notational simplicity, we take the same function $u_{1}(t)$ in (H3.2) and (H3.3).

(ii) We only consider the same dimensional case of $x$ and $p$. When $x$ and $p$ take different dimensions such as $x \in \mathbb{R}^{n}, p \in \mathbb{R}^{m}$, we can introduce a full rank $m \times n$ matrix and deal with it using the method in [17] to get the same result as the following Theorem 3.1.

THEOREM 3.1. We assume (H3.1), (H3.2) and (H3.3) hold, then FBSDEP (3.1) has a unique solution $(x(\cdot), p(\cdot), q(\cdot), k(\cdot)) \in \mathscr{S}^{2} \times \mathscr{S}^{2} \times \mathscr{H}^{2} \times F_{N}^{2}$.

Proof. For the uniqueness, let $u_{s}=\left(x_{s}, p_{s}, q_{s}, k_{s}\right)$ and $\bar{u}_{s}=\left(\bar{x}_{s}, \bar{p}_{s}, \bar{q}_{s}, \bar{k}_{s}\right)$ be two solutions of (3.1). We set $\hat{u}=(x-\bar{x}, p-\bar{p}, q-\bar{q}, k-\bar{k})=(\hat{x}, \hat{p}, \hat{q}, \hat{k})$ and apply Itô's formula to $\left\langle\hat{x}_{s}, \hat{p}_{s}\right\rangle$. Using the same technique, which was used to prove the uniqueness for FBSDE in [17], and the the uniqueness result for BSDEP and for stochastic differential equation with jump in [7], we can easily get the conclusion.

To prove the existence, we can consider two cases according to the signs of $\beta_{1}, \beta_{2}$ and $\mu_{1}$, this makes the proof clear and easy to understand.

First case. $\beta_{1}>0, \mu_{1}>0$ and $\beta_{2} \geq 0$.

We consider the following family of FBSDEP parametrized by $\alpha \in[0,1]$.

$$
\left\{\begin{aligned}
x_{t}^{\alpha}= & a+\int_{0}^{\imath \wedge \tau}\left[\alpha b\left(s, u_{s}^{\alpha}\right)+\phi_{s}\right] d s+\int_{0}^{\imath \wedge \tau}\left[\alpha \sigma\left(s, u_{s}^{\alpha}\right)+\psi_{s}\right] d B_{s} \\
\cdot & +\int_{0}^{\wedge \wedge \tau} \int_{z}\left[\alpha g\left(s_{-}, u_{s_{-}}^{\alpha}, z\right)+\lambda_{s_{-}}(z)\right] \tilde{N}(d z d s), \\
p_{t}^{\alpha}= & \alpha \Phi\left(x_{\tau}^{\alpha}\right)+(1-\alpha) x_{\tau}^{\alpha}+\xi+\int_{t \wedge \tau}^{\tau}\left[(1-\alpha) \beta_{1} u_{1}(s) x_{s}^{\alpha}+\alpha f\left(s, u_{s}^{\alpha}\right)+\gamma_{s}\right] d s \\
& -\int_{I^{\wedge} \tau \tau}^{\tau} q_{s}^{\alpha} d B_{s}-\int_{t \wedge \tau}^{\tau} \int_{Z} k_{s_{-}}^{\alpha}(z) \tilde{N}(d z d s),
\end{aligned}\right.
$$


where $\phi, \psi, \gamma$ and $\lambda$ are given processes with values in $\mathbb{R}^{m}, \mathbb{R}^{m \times d}, \mathbb{R}^{m}$ and $\mathbb{R}^{m}$ respectively, $\xi \in L^{2}$ and

$\mathbb{E}\left(\int_{0}^{\tau}\left|\phi_{s}\right| d s\right)^{2}+\mathbb{E}\left(\int_{0}^{\tau}\left|\gamma_{s}\right| d s\right)^{2}+\mathbb{E} \int_{0}^{\tau}\left|\psi_{s}\right|^{2} d s+\mathbb{E} \int_{0}^{\tau} \int_{Z}\left|\lambda_{s}(z)\right|^{2} n(d z) d s<\infty$.

Clearly, when $\alpha=1$, the existence of the solution of (3.2) implies this of (3.1). When $\alpha=0$, it is easy to see that there exists a solution of (3.2). So we need the following lemma.

LEMMA 3.2. We assume that (H3.1), (H3.2) and (H3.3) hold. Then there exists a positive constant $\delta_{0}$ such that if, apriorily, for $\alpha_{0} \in[0,1)$ there exists a solution $\left(x^{\alpha_{0}}, p^{\alpha_{0}}, q^{\alpha_{0}}, k^{\alpha_{0}}\right)$ of (3.2), then for each $\delta \in\left[0, \delta_{0}\right]$ there exists a solution $\left(x^{\alpha_{0}+\delta}, p^{\alpha_{0}+\delta}, q^{\alpha_{0}+\delta}, k^{\alpha_{0}+\delta}\right) \in \mathscr{S}^{2} \times \mathscr{S}^{2} \times \mathscr{H}^{2} \times F_{N}^{2}$ of (3.2) for $\alpha=\alpha_{0}+\delta$.

PROOF. Since for each $\phi, \gamma, \psi, \lambda, \alpha_{0} \in[0,1)$, there exists a solution of (3.2), then, for each triple

$$
u_{s}=\left(x_{s}, p_{s}, q_{s}, k_{s}\right) \in \mathscr{S}^{2} \times \mathscr{S}^{2} \times \mathscr{H}^{2} \times F_{N}^{2}, \quad x_{\tau} \in L^{2}
$$

there exists a unique triple $U_{s}=\left(X_{s}, P_{s}, Q_{s}, K_{s}\right) \in \mathscr{S}^{2} \times \mathscr{S}^{2} \times \mathscr{H}^{2} \times F_{N}^{2}$ satisfying the following FBSDEP

$$
\begin{aligned}
X_{t}= & a+\int_{0}^{t \wedge \tau}\left[\alpha_{0} b\left(s, U_{s}\right)+\delta b\left(s, u_{s}\right)+\phi_{s}\right] d s \\
& +\int_{0}^{t \wedge \tau}\left[\alpha_{0} \sigma\left(s, U_{s}\right)+\delta \sigma\left(s, u_{s}\right)+\psi_{s}\right] d B_{s} \\
& +\int_{0}^{t \wedge \tau} \int_{Z}\left[\alpha_{0} g\left(s_{-}, U_{s_{-}}, z\right)+\delta g\left(s_{-}, u_{s_{-}}, z\right)+\lambda_{s_{-}}\right] \tilde{N}(d z d s) \\
P_{t}= & \alpha_{0} \Phi\left(X_{\tau}\right)+\left(1-\alpha_{0}\right) X_{\tau}+\delta\left(\Phi\left(x_{\tau}\right)-x_{\tau}\right)+\xi \\
& +\int_{t \wedge \tau}^{\tau}\left[\left(1-\alpha_{0}\right) \beta_{1} u_{1}(s) X_{s}+\alpha_{0} f\left(s, U_{s}\right)+\delta\left(-\beta_{1} u_{1}(s) x_{s}+f\left(s, u_{s}\right)\right)+\gamma_{s}\right] d s \\
& -\int_{t \wedge \tau}^{\tau} Q_{s} d B_{s}-\int_{t \wedge \tau}^{\tau} \int_{Z} K_{s_{-}}(z) \tilde{N}(d z d s) .
\end{aligned}
$$

We want to prove that the mapping defined by

$$
\begin{aligned}
I_{\alpha_{0}+\delta}\left(u \times x_{\tau}\right) & =U \times X_{\tau}: \mathscr{S}^{2} \times \mathscr{S}^{2} \times \mathscr{H}^{2} \times F_{N}^{2} \times L^{2} \\
& \rightarrow \mathscr{S}^{2} \times \mathscr{S}^{2} \times \mathscr{H}^{2} \times F_{N}^{2} \times L^{2}
\end{aligned}
$$

is a contraction. 
We note that $\mathscr{B}^{2}=\mathscr{S}^{2} \times \mathscr{S}^{2} \times \mathscr{H}^{2} \times F_{N}^{2}$ and let $\bar{u}=(\bar{x}, \bar{p}, \bar{q}, \bar{k}) \in \mathscr{B}^{2}$, $\bar{U} \times \bar{X}_{\tau}=I_{\alpha_{0}+\delta}\left(\bar{u} \times \bar{x}_{\tau}\right)$. Using the same notations for $\hat{u}$ and $\widehat{U}$ as above and applying Itô's formula to $\left\langle\widehat{X}_{s}, \widehat{P}_{s}\right\rangle$, we get

$$
\begin{aligned}
{\left[\alpha_{0} \mu_{1}\right.} & \left.+\left(1-\alpha_{0}\right)\right] \mathbb{E}\left|\widehat{X}_{\tau}\right|^{2}+\beta_{1} \mathbb{E} \int_{0}^{\tau} u_{1}(s)\left|\widehat{X}_{s}\right|^{2} d s \\
\leq & \delta C_{1} \mathbb{E}\left|\hat{x}_{\tau}\right|^{2}+\delta C_{1} \mathbb{E}\left|\widehat{X}_{\tau}\right|^{2} \\
& +\delta C_{1}\left[\int_{0}^{\infty} u_{1}^{2}(s) d s+\left(\int_{0}^{\infty} u_{1}(s) d s\right)^{2}\right]\left[\|\widehat{U}(\cdot)\|_{\mathscr{B}^{2}}^{2}+\|\hat{u}(\cdot)\|_{\mathscr{B}^{2}}^{2}\right]
\end{aligned}
$$

Using Itô's formula to $\left|\widehat{P}_{s}\right|^{2}$ and then Gronwall's Lemma and the Burkholder-DavisGundy inequality, we get

$$
\begin{aligned}
\|\widehat{P}(\cdot)\|_{\mathscr{S}^{2}}^{2}+\|\widehat{Q}(\cdot)\|_{\mathscr{H}^{2}}^{2}+\|\widehat{K}(\cdot)\|_{F_{N}^{2}}^{2} \\
\leq C_{2}\left[\mathbb{E} \int_{0}^{\tau} u_{1}(s)\left|\widehat{X}_{s}\right|^{2} d s+\mathbb{E}\left|\widehat{X}_{\tau}\right|^{2}\right]+\delta C_{2} \mathbb{E}\left|\hat{x}_{\tau}\right|^{2} \\
\quad+\delta C_{2}\left[\int_{0}^{\infty} u_{1}^{2}(s) d s+\left(\int_{0}^{\infty} u_{1}(s) d s\right)^{2}\right]\|\hat{u}(\cdot)\|_{\mathscr{B}^{2}}^{2} .
\end{aligned}
$$

Applying the usual technique to the forward stochastic differential equation and combining with (3.3), we get

$$
\|\widehat{U}(\cdot)\|_{\mathscr{B}^{2}}^{2}+\left\|\widehat{X}_{\tau}\right\|_{L^{2}}^{2} \leq \delta M\left[\|\hat{u}(\cdot)\|_{\mathscr{B}^{2}}^{2}+\left\|\hat{x}_{\tau}\right\|_{L^{2}}^{2}\right] .
$$

Here the constants $C_{1}, C_{2}$ and $M$ depend on $\beta_{1}, \mu_{1}$ and $C$.

We now choose $\delta_{0}=1 /(2 M)$. It is clear that, for each fixed $\delta \in\left[0, \delta_{0}\right]$, the mapping $l_{\alpha_{0}+\delta}$ is a contraction and has a unique fixed point

$$
U^{\alpha_{0}+\delta}=\left(X^{\alpha_{0}+\delta}, P^{\alpha_{0}+\delta}, Q^{\alpha_{0}+\delta}, K^{\alpha_{0}+\delta}\right)
$$

which is the solution of (3.2) for $\alpha=\alpha_{0}+\delta$. The proof is complete.

Second case: $\beta_{2}>0, \beta_{1} \geq 0, \mu_{1} \geq 0$.

We need to consider the following family of FBSDEP parametrized by $\alpha \in[0,1]$.

$$
\left\{\begin{aligned}
x_{t}^{\alpha}= & a+\int_{0}^{t \wedge \tau}\left[\alpha b\left(s, u_{s}^{\alpha}\right)+(1-\alpha) \beta_{2}\left(-u_{1}(s) p_{s}^{\alpha}\right)+\phi_{s}\right] d s \\
& +\int_{0}^{1 \wedge \tau}\left[\alpha \sigma\left(s, u_{s}^{\alpha}\right)+(1-\alpha) \beta_{2}\left(-u_{1}(s) q_{s}^{\alpha}\right)+\psi_{s}\right] d B_{s} \\
& +\int_{0}^{t \wedge \tau} \int_{Z}\left[\alpha g\left(s_{-}, u_{s_{-}}^{\alpha}, z\right)+(1-\alpha) \beta_{2}\left(-u_{1}(s) k_{s_{-}}^{\alpha}\right)+\lambda_{s_{-}}(z)\right] \tilde{N}(d z d s) \\
p_{t}^{\alpha}= & \alpha \Phi\left(x_{\tau}^{\alpha}\right)+\xi+\int_{t \wedge \tau}^{\tau}\left[\alpha f\left(s, u_{s}^{\alpha}\right)+\gamma_{s}\right] d s-\int_{t \wedge \tau}^{\tau} q_{s}^{\alpha} d B_{s}-\int_{t \wedge \tau}^{\tau} k_{s-}^{\alpha}(z) \widetilde{N}(d z d s),
\end{aligned}\right.
$$


where $\phi, \psi, \gamma, \lambda$ and $\xi$ satisfy the same assumptions as that in (3.2). Similarly to Lemma 3.2, we can show the following result.

LEMMA 3.3. We assume (H3.1), (H3.2) and (H3.3) hold, then there exists a positive constant $\delta_{0}$ such that if, apriorily, for an $\alpha_{0} \in[0,1)$ there exists a solution $\left(x^{\alpha_{0}}, p^{\alpha_{0}}, q^{\alpha_{0}}, k^{\alpha_{0}}\right)$ of (3.4), then for each $\delta \in\left[0, \delta_{0}\right]$ there exists a solution $\left(x^{\alpha_{0}+\delta}, p^{\alpha_{0}+\delta}, q^{\alpha_{0}+\delta}, k^{\alpha_{0}+\delta}\right) \in \mathscr{S}^{2} \times \mathscr{S}^{2} \times \mathscr{H}^{2} \times F_{N}^{2}$ of (3.4) for $\alpha=\alpha_{0}+\delta$.

PROOF OF THEOREM 3.1 (EXISTENCE). From the assumption (H3.3), we know that either (i) $\beta_{1}>0, \mu_{1}>0, \beta_{2} \geq 0$ or (ii) $\beta_{1} \geq 0, \mu_{1} \geq 0, \beta_{2}>0$. In the first case, we consider (3.2) and when $\alpha=0$, (3.2) has a unique solution. It then follows from Lemma 3.2 that there exists a positive constant $\delta_{0}$ such that for each $\delta \in\left[0, \delta_{0}\right]$, (3.2) has a unique solution for $\alpha=\alpha_{0}+\delta$. We can repeat this process $N$-times with $1 \leq N \delta_{0}<1+\delta_{0}$. It then follows that, in particular, for $\alpha=1$ with $\phi_{s}=0, \gamma_{s}=0$, $\psi_{s}=0, \lambda_{s}=0$ and $\xi=0$ (3.2) has a unique solution.

In the second case, we consider (3.4) and when $\alpha=0, \operatorname{FBSDEP}(3.4)$ has a unique solution. It then follows from Lemma 3.3 , by repeating the same process as in the first case, that we get the desired conclusion. The proof is completed.

REMARK 3.2. If we replace (H3.3) by the following (H3.4) For every $u=(x, p, q, k), \bar{u}=(\bar{x}, \bar{p}, \bar{q}, \bar{k}), \hat{u}=(\hat{x}, \hat{p}, \hat{q}, \hat{k})=(x-\bar{x}$, $p-\bar{p}, q-\bar{q}, k-\bar{k})$,

$$
\left\{\begin{aligned}
\langle A(t, u)-A(t, \bar{u}), \hat{u}\rangle & \geq \beta_{1} u_{1}(t)|\hat{x}|^{2}+\beta_{2} u_{1}(t)\left(|\hat{p}|^{2}+|\hat{q}|^{2}+|\hat{k}|^{2}\right) \\
\langle\Phi(x)-\Phi(\bar{x}), x-\bar{x}\rangle & \leq-\mu_{1}|\hat{x}|^{2},
\end{aligned}\right.
$$

where $\beta_{1}, \beta_{2}$ and $\mu_{1}$ are given nonnegative constants with $\beta_{1}+\beta_{2}>0, \mu_{1}+\beta_{2}>0$. Using a similar method as in Theorem 3.1, we can also prove that FBSDEP (3.1) has the unique solution.

REMARK 3.3. When the stopping time $\tau \leq T<\infty, u_{1}(t), 0 \leq t \leq T$, can be replaced by the constant, then the existence and uniqueness result of FBSDEP in bounded time duration is the special case of Theorem 3.1 .

\section{The comparison theorem of FBSDEP in stopping time duration}

In this section, we give a comparison theorem to FBSDEP in stopping time. This theorem is one of important properties of FBSDEP. We consider the following two 
FBSDEPs,

$$
\left\{\begin{aligned}
x_{t}^{i}= & a^{i}+\int_{0}^{t \wedge \tau} b\left(s, x_{s}^{i}, p_{s}^{i}, q_{s}^{i}\right) d s+\int_{0}^{i \wedge \tau} \sigma\left(s, x_{s}^{i}, p_{s}^{i}, q_{s}^{i}\right) d B_{s} \\
& +\int_{0}^{i \wedge \tau} \int_{Z} g\left(s_{-}, x_{s_{-}}^{i}, p_{s_{-}}^{i}, q_{s_{-}}^{i}, z\right) \tilde{N}(d z d s), \quad i=1,2, \\
p_{t}^{i}= & \Phi^{i}\left(x_{\tau}^{i}\right)+\int_{t \wedge \tau}^{\tau} f^{i}\left(s, x_{s}^{i}, p_{s}^{i}, q_{s}^{i}, k_{s}^{i}\right) d s-\int_{t \wedge \tau}^{\tau} q_{s}^{i} d B_{s} \\
& -\int_{0}^{l \wedge \tau} \int_{Z} k_{s_{-}}^{i} \tilde{N}(d z d s) .
\end{aligned}\right.
$$

The coefficients of FBSDEP (4.1), $i=1,2$, both satisfy (H3.1), (H3.2) and (H3.3), then there exists the solution $\left(x^{i}, p^{i}, q^{i}, k^{i}\right) \in \mathscr{S}^{2} \times \mathscr{S}^{2} \times \mathscr{H}^{2} \times F_{N}^{2}$ respectively. In the following part, we only consider $m=1$, in fact we can also deal with the case when $x$ takes multidimensional value such as $x \in \mathbb{R}^{n}$. For that case, we need to introduce a $1 \times n$ nonzero vector $G$ in the monotone assumptions to ensure the existence and uniqueness for different dimensional FBSDEP the same as that in [17].

We assume

(H4.1) For every $x \in \mathbb{R}, s \geq 0$,

$$
\left\{\begin{array}{l}
a^{1} \geq a^{2}, \quad \Phi^{1}(x) \geq \Phi^{2}(x), \quad \text { a.s. } \\
f^{1}(s, x, p, q, k) \geq f^{2}(s, x, p, q, k), \quad \text { a.s. }
\end{array}\right.
$$

The introduction of a random jump let the solutions $x$ and $p$ to be not continuous, so we also need the following condition to control the jump height.

$$
\text { (H4.2) }-1<\frac{f^{1}\left(s, x_{s}^{2}, p_{s}^{2}, q_{s}^{2}, k_{s}^{1}\right)-f^{1}\left(s, x_{s}^{2}, p_{s}^{2}, q_{s}^{2}, k_{s}^{2}\right)}{k_{s}^{1}-k_{s}^{2}}, k_{s}^{1}-k_{s}^{2} \neq 0 \text {, a.s. }
$$

Then we have

THEOREM 4.1. $p_{0}^{1} \geq p_{0}^{2}$.

ProOF. For notational convenience, we assume $d=1$ and first consider the following FBSDEP:

$$
\left\{\begin{aligned}
\bar{x}_{t}= & a^{1}+\int_{0}^{i \wedge \tau} b\left(s, \bar{x}_{s}, \bar{p}_{s}, \bar{q}_{s}\right) d s+\int_{0}^{i \wedge \tau} \sigma\left(s, \bar{x}_{s}, \bar{p}_{s}, \bar{q}_{s}\right) d B_{s} \\
& +\int_{0}^{i \wedge \tau} \int_{Z} g\left(s_{-}, \bar{x}_{s_{-}}, \bar{p}_{s_{-}}, \bar{q}_{s_{-}}, z\right) \tilde{N}(d z d s), \quad i=1,2, \\
\bar{p}_{t}= & \Phi^{2}\left(\bar{x}_{\tau}\right)+\int_{t \wedge \tau}^{\tau} f^{2}\left(s, \bar{x}_{s}, \bar{p}_{s}, \bar{q}_{s}, \bar{k}_{s}\right) d s-\int_{t \wedge \tau}^{\tau} \bar{q}_{s} d B_{s} \\
& -\int_{0}^{i \wedge \tau} \int_{Z} \bar{k}_{s_{-}} \tilde{N}(d z d s) .
\end{aligned}\right.
$$


Obviously, the above FBSDEP has a unique solution $(\bar{x}, \bar{p}, \bar{q}, \bar{k})$. We set $\hat{x}=x^{1}-\bar{x}$, $\hat{p}=p^{1}-\bar{p}, \hat{q}=q^{1}-\bar{q}, \hat{k}=k^{1}-\bar{k}$, the quartet $(\hat{x}, \hat{p}, \hat{q}, \hat{k})$ satisfies

$$
\left\{\begin{aligned}
\hat{x}_{t}= & \int_{0}^{t \wedge \tau}\left(b_{s}^{1} \hat{x}_{s}+b_{s}^{2} \hat{p}_{s}+b_{s}^{3} \hat{q}_{s}\right) d s+\int_{0}^{t \wedge \tau}\left(\sigma_{s}^{1} \hat{x}_{s}+\sigma_{s}^{2} \hat{p}_{s}+\sigma_{s}^{3} \hat{q}_{s}\right) d B_{s} \\
& +\int_{0}^{t \wedge \tau} \int_{z}\left(g_{s_{-}}^{1} \hat{x}_{s_{-}}+g_{s_{-}}^{2} \hat{p}_{s_{-}}+g_{s_{-}}^{3} \hat{q}_{s_{-}}\right) \tilde{N}(d z d s) \\
\hat{p}_{t}= & \bar{\Phi} \hat{x}_{\tau}+\Phi^{1}\left(\bar{x}_{\tau}\right)-\Phi^{2}\left(\bar{x}_{\tau}\right)+\int_{t \wedge \tau}^{\tau}\left(f_{s}^{11} \hat{x}_{s}+f_{s}^{12} \hat{p}_{s}+f_{s}^{13} \hat{q}_{s}\right. \\
& \left.+f_{s}^{14} \hat{k}_{s}+\overline{f_{s}}\right) d s-\int_{t \wedge \tau}^{\tau} \hat{q}_{s} d B_{s}-\int_{t \wedge \tau}^{\tau} \int_{Z} \hat{k}_{s_{-}}(z) \tilde{N}(d z d s),
\end{aligned}\right.
$$

where $\overline{f_{s}}=f^{1}(s, \bar{x}, \bar{p}, \bar{q}, \bar{k})-f^{2}(s, \bar{x}, \bar{p}, \bar{q}, \bar{k})$,

$$
\begin{aligned}
& \bar{\Phi}= \begin{cases}\frac{\Phi^{1}\left(x_{\tau}^{1}\right)-\Phi^{1}\left(\bar{x}_{\tau}\right)}{x_{\tau}^{1}-\bar{x}_{\tau}}, & \hat{x}_{\tau} \neq 0, \\
0, & \text { otherwise, }\end{cases} \\
& l_{s}^{1}= \begin{cases}\frac{l\left(s, x_{s}^{1}, p_{s}^{1}, q_{s}^{1}\right)-l\left(s, \bar{x}_{s}, p_{s}^{1}, q_{s}^{1}\right)}{x_{s}^{1}-\bar{x}_{s}}, & \hat{x}_{s} \neq 0, \\
0, & \text { otherwise, }\end{cases} \\
& l_{s}^{2}= \begin{cases}\frac{l\left(s, \bar{x}_{s}, p_{s}^{1}, q_{s}^{1}\right)-l\left(s, \bar{x}_{s}, \bar{p}_{s}, q_{s}^{1}\right)}{p_{s}^{1}-\bar{p}_{s}}, & \hat{p}_{s} \neq 0, \\
0, & \text { otherwise, }\end{cases} \\
& l_{s}^{3}= \begin{cases}\frac{l\left(s, \bar{x}_{s}, \bar{p}_{s}, q_{s}^{1}\right)-l\left(s, \bar{x}_{s}, \bar{p}_{s}, \bar{q}_{s}\right)}{q_{s}^{1}-\bar{q}_{s},} & \hat{q}_{s} \neq 0, \\
0, & \text { otherwise }\end{cases}
\end{aligned}
$$

$l=b, \sigma, g$ respectively.

$$
\begin{aligned}
& f_{s}^{11}= \begin{cases}\frac{f^{1}\left(s, x_{s}^{1}, p_{s}^{1}, q_{s}^{1}, k_{s}^{1}\right)-f^{1}\left(s, \bar{x}_{s}, p_{s}^{1}, q_{s}^{1}, k_{s}^{1}\right)}{x_{s}^{1}-\bar{x}_{s}}, & \hat{x}_{s} \neq 0, \\
0, & \text { otherwise, }\end{cases} \\
& f_{s}^{12}= \begin{cases}\frac{f^{1}\left(s, \bar{x}_{s}, p_{s}^{1}, q_{s}^{1}, k_{s}^{1}\right)-f^{1}\left(s, \bar{x}_{s}, \bar{p}_{s}, q_{s}^{1}, k_{s}^{1}\right)}{p_{s}^{1}-\bar{p}_{s}}, & \hat{p}_{s} \neq 0, \\
0, & \text { otherwise }\end{cases} \\
& f_{s}^{13}= \begin{cases}\frac{f^{1}\left(s, \bar{x}_{s}, \bar{p}_{s}, q_{s}^{1}, k_{s}^{1}\right)-f^{1}\left(s, \bar{x}_{s}, \bar{p}_{s}, \bar{q}_{s}, k_{s}^{1}\right)}{q_{s}^{1}-\bar{q}_{s}}, & \hat{q}_{s} \neq 0, \\
0, & \text { otherwise, }\end{cases}
\end{aligned}
$$




$$
f_{s}^{14}= \begin{cases}\frac{f^{1}\left(s, \bar{x}_{s}, \bar{p}_{s}, \bar{q}_{s}, k_{s}^{1}\right)-f^{1}\left(s, \bar{x}_{s}, \bar{p}_{s}, \bar{q}_{s}, \bar{k}_{s}\right)}{k_{s}^{1}-\bar{k}_{s}}, & \hat{k}_{s} \neq 0 \\ 0, & \text { otherwise }\end{cases}
$$

It is easy to check that (4.3) satisfies (H3.1), (H3.2) and (H3.3), thus $(\hat{x}, \hat{p}, \hat{q}, \hat{k})$ is the unique solution of (4.3). We first need to prove that $\hat{p}_{0} \geq 0$. We use the duality technique and introduce the dual FBSDEP

$$
\left\{\begin{aligned}
M_{t}= & 1+\int_{0}^{t \wedge \tau}\left(f_{s}^{12} M_{s}-b_{s}^{2} N_{s}-\sigma_{s}^{2} U_{s}-g_{s}^{2} V_{s}\right) d s \\
& \left.+\int_{0}^{t \wedge \tau} f_{s}^{13} M_{s}-b_{s}^{3} N_{s}-\sigma_{s}^{3} U_{s}-g_{s}^{3} V_{s}\right) d B_{s} \\
& +\int_{0}^{t \wedge \tau} \int_{Z} f_{s}^{14} M_{s-} \tilde{N}(d z d s), \\
N_{t}= & -\bar{\Phi} M_{\tau}+\int_{t \wedge \tau}^{\tau}\left(-f_{s}^{1} M_{s}+b_{s}^{1} N_{s}+\sigma_{s}^{1} U_{s}+g_{s}^{1} V_{s}\right) d s \\
& -\int_{t \wedge \tau}^{\tau} U_{s} d B_{s}-\int_{t \wedge \tau}^{\tau} \int_{Z} V_{s-}(z) \tilde{N}(d z d s) .
\end{aligned}\right.
$$

The duality technique is usually used to introduce the adjoint equation in optimal control theory when we want to get the maximum principle (see [14] and [20]). From (4.3) satisfying (H3.1), (H3.2) and (H3.3), we can verify that (4.4) satisfies (H3.1), (H3.2) and (H3.4). Then it follows from Remark 3.2 that there exists a unique quartet $(M, N, U, V)$ which is the solution of (4.4).

Applying Itô's formula to $\hat{x}_{s} N_{s}+\hat{p}_{s} M_{s}$, we have

$$
\hat{p}_{0}=\mathbb{E}\left(\Phi^{1}\left(\bar{x}_{\tau}\right)-\Phi^{2}\left(\bar{x}_{\tau}\right)\right) M_{\tau}+\mathbb{E} \int_{0}^{\tau} M_{s} \overline{f_{s}} d s .
$$

From (H4.1) and $M_{0}=1>0$, if we can prove $M_{s \wedge \tau} \geq 0$, a.s. $s \geq 0$, then $\hat{p}_{0} \geq 0$.

Let us define the following stopping time

$$
\nu=\inf \left\{t>0 ; M_{t} \leq 0\right\} \wedge \tau \text {. }
$$

So $\nu \leq \tau$, a.s. and $M_{\nu_{-}} \geq 0$. In the first equation of (4.4), the noncontinuous part of $M_{t}$ is only produced by random measure $N$, from (H4.2),

$$
\Delta M_{v} \geq-M_{v_{-}}, \quad M_{v}=M_{v_{-}}+\Delta M_{v} \geq 0,
$$

so $M_{\nu}=0$, when $\nu<\tau$. We can introduce $\left(\bar{M}_{t}, \bar{N}_{t}, \bar{U}_{t}, \bar{V}_{t}\right), t \in[\nu, \tau]$, which satisfies 
the following FBSDEP

$$
\left\{\begin{aligned}
\bar{M}_{t}= & \int_{v}^{t \wedge \tau}\left(f_{s}^{12} \bar{M}_{s}-b_{s}^{2} \bar{N}_{s}-\sigma_{s}^{2} \bar{U}_{s}-g_{s}^{2} \bar{V}_{s}\right) d s \\
& +\int_{v}^{t \wedge \tau}\left(f_{s}^{13} \bar{M}_{s}-b_{s}^{3} \bar{N}_{s}-\sigma_{s}^{3} \bar{U}_{s}-g_{s}^{3} \bar{V}_{s}\right) d B_{s} \\
& +\int_{v}^{t \wedge \tau} \int_{Z} f_{s}^{14} \bar{M}_{s-} \tilde{N}(d z d s) \\
\bar{N}_{t}= & -\bar{\Phi} \bar{M}_{\tau}+\int_{t \wedge \tau}^{\tau}\left(-f_{s}^{1} \bar{M}_{s}+b_{s}^{1} \bar{N}_{s}+\sigma_{s}^{1} \bar{U}_{s}+g_{s}^{1} \bar{V}_{s}\right) d s \\
& -\int_{t \wedge \tau}^{\tau} \bar{U}_{s} d B_{s}-\int_{t \wedge \tau}^{\tau} \int_{Z} \bar{V}_{s-}(z) \tilde{N}(d z d s) .
\end{aligned}\right.
$$

Then it is easy to see that $\left(\bar{M}_{t}, \bar{N}_{t}, \bar{U}_{t}, \bar{V}_{t}\right) \equiv(0,0,0,0)$ is the unique solution. Now we let

$$
\begin{aligned}
M_{t}^{\prime} & =1_{[0, v]}(t) M_{t}+1_{(v, \tau]}(t) \bar{M}_{t}, & N_{t}^{\prime}=1_{[0, v]}(t) N_{t}+1_{(v, \tau]}(t) \bar{N}_{t} \\
U_{t}^{\prime} & =1_{[0, v]}(t) U_{t}+1_{(v, \tau]}(t) \bar{U}_{t}, & V_{t}^{\prime}=1_{[0, v]}(t) V_{t}+1_{(v, \tau]}(t) \bar{V}_{t}, \quad 0 \leq t \leq \tau .
\end{aligned}
$$

It is easy to see that $\left(M_{t}^{\prime}, N_{t}^{\prime}, U_{t}^{\prime}, V_{t}^{\prime}\right)$ is a solution of (4.4), from Remark 3.2, this is the unique solution. From $M_{0}^{\prime}=M_{0}=1>0$ and $M_{\nu} \geq 0$, obviously $M_{s \wedge \tau}^{\prime} \geq 0$, a.s. $s \geq 0$, that is, $M_{s \wedge \tau} \geq 0$. So we have $p_{0}^{1} \geq \bar{p}_{0}$.

Now we try to compare $\bar{p}_{0}$ with $p_{0}^{2}$, and then get the desired conclusion. If $a^{1}=a^{2}$, from Theorem 3.1, $\bar{p}_{0}=p_{0}^{2}$, then $p_{0}^{1} \geq p_{0}^{2}$. If $a^{1}>a^{2}$, we set

$$
\begin{aligned}
& \tilde{x}=\left(\bar{x}-x^{2}\right), \quad \tilde{p}=\left(\bar{p}-p^{2}\right), \quad \tilde{u}=\left(\bar{u}-u^{2}\right) \\
& \tilde{q}=\left(\bar{q}-q^{2}\right), \quad \tilde{k}=\left(\bar{k}-k^{2}\right),
\end{aligned}
$$

and apply Itô's formula to $\tilde{x}_{t} \tilde{p}_{t}$,

$$
\mathbb{E}\left(\Phi\left(\bar{x}_{\tau}\right)-\Phi\left(x_{\tau}^{2}\right)\right) \tilde{x}_{\tau}-\left(\bar{p}_{0}-p_{0}^{2}\right)\left(a^{1}-a^{2}\right)=\mathbb{E} \int_{0}^{\tau}\left\langle A\left(s, \bar{u}_{s}\right)-A\left(s, u_{s}^{2}\right), \tilde{u}_{s}\right\rangle d s .
$$

Here we use the notation from Section 3 for $u$ and $A$. From (H3.3), we have

$$
\left(\bar{p}_{0}-p_{0}^{2}\right)\left(a^{1}-a^{2}\right) \geq 0,
$$

so $\overline{p_{0}} \geq p_{0}^{2}$, and then $p_{0}^{1} \geq p_{0}^{2}$. The proof is completed.

Now we give an example of FBSDEP to show the comparison theorem. 
EXAMPLE 4.1. We consider the following two FBSDEPs,

$$
\left\{\begin{aligned}
x_{t}^{1}= & 2-\int_{0}^{t \wedge \tau} \frac{p_{s}^{1}+x_{s}^{1}+q_{s}^{1}}{(1+s)^{2}} d s-\int_{0}^{t \wedge \tau} \frac{q_{s}^{1}-p_{s}^{1}}{(1+s)^{2}} d B_{s} \\
& -\int_{0}^{t \wedge \tau} \int_{Z} \frac{x_{s}^{1}}{(1+s)^{2}} \tilde{N}(d z d s), \\
p_{t}^{1}= & x_{\tau}^{1}+5+\int_{t \wedge \tau}^{\tau}\left(\frac{x_{s}^{1}-p_{s}^{1}-k_{s}^{1}}{(1+s)^{2}}+\frac{1}{(1+s)^{2}}\right) d s \\
& -\int_{t \wedge \tau}^{\tau} q_{s}^{1} d B_{s}-\int_{t \wedge \tau}^{\tau} \int_{Z} k_{s_{-}}^{1}(z) \tilde{N}(d z d s), \quad t \geq 0
\end{aligned}\right.
$$

and

$$
\left\{\begin{aligned}
x_{t}^{2}= & 1-\int_{0}^{\imath \wedge \tau} \frac{p_{s}^{2}+x_{s}^{2}+q_{s}^{2}}{(1+s)^{2}} d s-\int_{0}^{t \wedge \tau} \frac{q_{s}^{2}-p_{s}^{2}}{(1+s)^{2}} d B_{s} \\
& -\int_{0}^{i \wedge \tau} \int_{Z} \frac{x_{s}^{2}}{(1+s)^{2}} \tilde{N}(d z d s), \\
p_{t}^{2}= & x_{\tau}^{2}+\int_{t \wedge \tau}^{\tau} \frac{x_{s}^{2}-p_{s}^{2}-k_{s}^{2}}{(1+s)^{2}} d s \\
& -\int_{t \wedge \tau}^{\tau} q_{s}^{2} d B_{s}-\int_{t \wedge \tau}^{\tau} \int_{Z} k_{s-}^{2}(z) \tilde{N}(d z d s), \quad t \geq 0 .
\end{aligned}\right.
$$

It is easy to check that (4.6) and (4.7) satisfy (H3.1), (H3.2) and (H3.3), so according to Theorem 3.1, there exist unique solutions $\left(x^{1}, p^{1}, q^{1}, k^{1}\right)$ and $\left(x^{2}, p^{2}, q^{2}, k^{2}\right)$ respectively. We can check that the above two FBSDEPs satisfy (H4.1) and (H4.2), so from Theorem 4.1, we know that $p_{0}^{1} \geq p_{0}^{2}$.

We notice that the comparison Theorem 4.1 of FBSDEP, which holds only at time $t=0$, is weaker than that of BSDEP, that is, Theorem 2.2. In the forward-backward case, we cannot easily jump to a conclusion like $p_{\tau}^{1}=\Phi^{1}\left(x_{\tau}^{1}\right) \geq \Phi^{2}\left(x_{\tau}^{2}\right)=p_{\tau}^{2}$ from the assumption that $\Phi^{1}(x) \geq \Phi^{2}(x)$ because in the present situation, the forward solutions $x_{\mathfrak{r}}^{1}$ and $x_{\tau}^{2}$ are different if $\Phi^{1}$ and $\Phi^{2}$ are. Thus unlike the classical (pure backward) case, no common comparison theorem can be made even $a^{1}=a^{2}$ except for $t=0$. We will give a counterexample to show this point.

EXAMPLE 4.2. For simplicity, we consider the fixed time duration $T>0$, the Lipschitz coefficient being constant, a one dimensional Brownian motion and study the following two FBSDEPs,

$$
\left\{\begin{array}{l}
x_{t}^{1}=a+\int_{0}^{t}\left(-p_{s}^{1}+q_{s}^{1}\right) d s-\int_{0}^{t}\left(x_{s}^{1}+p_{s}^{1}+q_{s}^{1}\right) d B_{s}, \quad 0 \leq t \leq T, \\
p_{t}^{1}=x_{T}^{1}+2+\int_{t}^{T}\left(x_{s}^{1}-q_{s}^{1}+2\right) d s-\int_{t}^{T} q_{s}^{1} d B_{s}-\int_{t}^{T} \int_{Z} k_{s_{-}}^{1}(z) \tilde{N}(d z d s),
\end{array}\right.
$$




$$
\left\{\begin{array}{l}
x_{t}^{2}=a+\int_{0}^{t}\left(-p_{s}^{2}+q_{s}^{2}\right) d s-\int_{0}^{t}\left(x_{s}^{2}+p_{s}^{2}+q_{s}^{2}\right) d B_{s}, \quad 0 \leq t \leq T, \\
p_{t}^{2}=x_{T}^{2}+\int_{t}^{T}\left(x_{s}^{2}-q_{s}^{2}\right) d s-\int_{t}^{T} q_{s}^{2} d B_{s}-\int_{t}^{T} \int_{Z} k_{s_{-}}^{2}(z) \tilde{N}(d z d s),
\end{array}\right.
$$

From Theorem 3.1 and Remark 3.3, there exist a unique solution $\left(x^{1}, p^{1}, q^{1}, k^{1}\right)$ for (4.8) and $\left(x^{2}, p^{2}, q^{2}, k^{2}\right)$ for (4.9) respectively. Then, from Theorem $4.1, p_{0}^{2} \geq p_{0}^{1}$. Now we try to check this conclusion for this example.

Firstly, it is easy to know that $\left(x^{1}, p^{1}, q^{1}, k^{1}\right)$ is the unique solution of (4.8), where $p_{t}^{1}=x_{t}^{1}+2, q_{t}^{1}=-x_{t}^{1}-1, k_{t}^{1}=0$ and $x_{t}^{1}$ is the solution of the following stochastic differential equation:

$$
\left\{\begin{array}{l}
d x_{t}^{1}=\left(-2 x_{t}^{1}-3\right) d t-\left(x_{t}^{1}+1\right) d B_{t} \\
x_{0}^{1}=a
\end{array}\right.
$$

Then we get

$$
x_{t}^{1}=a e^{-5 t / 2-B_{t}}-e^{-5 t / 2-B_{t}} \int_{0}^{t} 4 e^{5 s / 2+B_{s}} d s-e^{-5 t / 2-B_{t}} \int_{0}^{t} e^{5 s / 2+B_{s}} d B_{s}
$$

and $p_{t}^{1}=x_{t}^{1}+2,0 \leq t \leq T$. We also can get $\left(x^{2}, p^{2}, q^{2}, k^{2}\right)$ is the unique solution of (4.9), where $p_{t}^{2}=x_{t}^{2}, q_{t}^{2}=-x_{t}^{2}, k_{t}^{2}=0$ and $x_{t}^{2}$ satisfies the following stochastic differential equation

$$
\left\{\begin{array}{l}
d x_{t}^{2}=-2 x_{t}^{2} d t-x_{t}^{2} d B_{t} \\
x_{0}^{2}=a
\end{array}\right.
$$

Then $p_{t}^{2}=x_{t}^{2}=a e^{-5 t / 2-B_{t}}$. So

$$
p_{t}^{1}-p_{t}^{2}=2-e^{-5 t / 2-B_{t}} \int_{0}^{t} 4 e^{5 s / 2+B_{s}} d s-e^{-5 t / 2-B_{t}} \int_{0}^{t} e^{5 s / 2+B_{s}} d B_{s} .
$$

For $t=0, p_{0}^{1}-p_{0}^{2}=2>0$, but for any $t>0$, it can be both positive or negative with positive probability.

Acknowledgement The author thanks the referee for his many helpful comments and suggestions.

\section{References}

[1] F. Antonelli, 'Backward-forward stochastic differential equations', Ann. Appl. Probab. 3 (1993), 777-793. 
[2] G. Barles, R. Buckdahn and E. Pardoux, 'Backward stochastic differential equations and integralpartial differential equations', Stochastics Stochastics Rep. 60 (1997), 57-83.

[3] R. Darling and E. Pardoux, 'Backward SDE with random terminal time and applications to semilinear elliptic PDE', Ann. Probab. 25 (1997), 1135-1159.

[4] D. Duffie and L. Epstein, 'Stochastic differential utilities', Econometrica 60 (1992), 354-439.

[5] S. Hamadène, 'Backward-forward SDE's and stochastic differential games', Stochastic Process Appl. 77 (1998), 1-15.

[6] Y. Hu and S. Peng, 'Solution of forward-backward stochastic differential equations', Probab. Theory Related Fields 103 (1995), 273-283.

[7] N. Ikeda and S. Watanabe, Stochastic differential equations and diffusion processes (Nauka, Moscow, 1986).

[8] N. El Karoui, S. Peng and M.-C. Quenez, 'Backward stochastic differential equation in finance', Math. Finance 7 (1997), 1-71.

[9] J. Ma, P. Protter and J. Yong, 'Solving forward-backward stochastic differential equations explicitly - a four step scheme', Probab. Theory Related Fields 98 (1994), 339-359.

[10] E. Pardoux, 'Generalized discontinuous backward stochastic differential equations', in: Backward stochastic differential equations (eds. El Karoui and L. Mazliak), Pitman Res. Notes Math. Ser. 364 (Longman, Harlow, 1997) pp. 207-219.

[11] E. Pardoux and S. Peng, 'Adapted solution of a backward stochastic differential equation', Systems Control Lett. 14 (1990), 55-61.

[12] E. Pardoux and S. Tang, 'Forward-backward stochastic differential equations and quasi-linear parabolic PDEs', Probab. Theory Related Fields 114 (1999), 123-150.

[13] S. Peng, 'Probabilistic interpretation for systems of quasilinear parabolic partial differential equations', Stochastics Stochastics Rep. 37 (1991), 61-74.

[14] - 'Backward stochastic differential equations and applications to optimal control', Appl. Math. Optim. 27 (1993), 125-144.

[15] —_, 'Open problems on backward stochastic differential equations', in: Control of distributed parameter and stochastic systems (eds. S. Chen, X. Li, J. Yong and X. Y. Zhou) (Kluwer, Boston, 1999) pp. 265-273.

[16] S. Peng and Y. Shi, 'Infinite horizon forward-backward stochastic differential equations', Stochastic Process Appl. 85 (2000), 75-92.

[17] S. Peng and Z. Wu, 'Fully coupled forward-backward stochastic differential equations and applications to optimal control', SIAM J. Control Optim. 37 (1999), 825-843.

[18] R. Situ, 'On solution of backward stochastic differential equations with jumps and applications', Stochastic Process Appl. 66 (1997), 209-236.

[19] S. Tang and X. Li, 'Necessary condition for optimal control of stochastic systems with random jumps', SIAM J. Control Optim. 32 (1994), 1447-1475.

[20] W. Xu, 'Stochastic maximum principle for optimal control problem of forward and backward system', J. Austral. Math. Soc. Ser. B 37 (1995), 172-185.

[21] J. Yong, 'Finding adapted solution of forward-backward stochastic differential equations-method of continuation', Probab. Theory Related Fields 107 (1997), 537-572.

School of Mathematics and System Science

Shandong University

Jinan 250100

China

e-mail: wuzhen@sdu.edu.cn 